IHES/P $/ 05 / 15$

hep-th/0505068

\title{
Gauge invariants and Killing tensors in higher-spin gauge theories
}

\author{
Xavier Bekaert ${ }^{\star 1}$ and Nicolas Boulanger ${ }^{\star 2}$ \\ * Institut des Hautes Études Scientifiques, Le Bois-Marie \\ 35 route de Chartres, 91440 Bures-sur-Yvette (France) \\ `Université de Mons-Hainaut, Mécanique et Gravitation \\ 6 Avenue du Champ de Mars, 7000 Mons (Belgium)
}

\begin{abstract}
In free completely symmetric tensor gauge field theories on Minkowski space-time, all gauge invariant functions and Killing tensor fields are computed, both on-shell and off-shell. These problems are addressed in the metric-like formalisms.
\end{abstract}

PACS codes: 11.30.Ly, 02.20.Tw, 11.10.Kk

Keywords: Higher-spins, Killing tensors, BRST

\footnotetext{
${ }^{1}$ E-mail address: bekaert@ihes.fr

${ }^{2}$ Chargé de Recherches FNRS (Belgium), E-mail address: nicolas.boulanger@umh.ac.be
} 


\section{Introduction}

The old Fronsdal's programme - that consists in introducing consistent interactions among massless higher-spin fields [1] - still remains an important open mathematical problem of classical field theory. Numerous preliminary results toward this goal have been obtained (see e.g. the review paper [2] and references therein) which reveal surprising properties of higher-spin gauge fields. A better ("geometrical") understanding of the gauge structure underlying these theories will presumably be necessary for actually completing Fronsdal's programme. As a very preliminary step in this direction, some properties of the Abelian gauge transformations of the free theories are investigated closely in the present paper. More precisely, we focus on the following problems: the determination of all gauge invariant functions and all Killing tensor fields of free completely symmetric tensor gauge field theories in flat background, respectively on-shell and off-shell, under the sole assumption of locality. These problems are solved in both constrained and unconstrained metric-like approaches.

The plan of the paper is as follows. Free higher-spin gauge theories are reviewed in Section 2 where the problems addressed are formulated in a more precise way. As explained in Section 3, the problems are solved via a cohomological reformulation in the Becchi-RouetStora-Tyutin (BRST) formalism and their general solutions are contained in the corollary of Theorem 1 and in Theorem 2 which are given in the section 4 that concludes the paper. In order to spell out the notation and make the paper as self-contained as possible, Appendix A reviews some textbook material on irreducible representations of the general linear and orthogonal Lie algebras. Theorems 1 and 2 are respectively proved in Appendices B and C.

\section{Statement of the problems}

Einstein's gravity theory is a non-Abelian massless spin-2 field theory, the two main formulations of which are the "metric" and the "frame" approaches. In a very close analogy, there exist two main approaches to higher-spin (i.e. spin $s>2$ ) field theories that are by-now referred to as "metric-like" [1,3] and "frame-like" [2, 4]. The present paper essentially deals with the former approach where the massless field is represented by a completely symmetric field $\varphi$ of $\operatorname{rank}^{3} s>0$, the gauge transformation of which reads

$$
\delta_{\varepsilon} \varphi_{\mu_{1} \ldots \mu_{s}}=s \partial_{\left(\mu_{1}\right.} \varepsilon_{\left.\mu_{2} \ldots \mu_{s}\right)}
$$

where the curved bracket denotes complete symmetrization with strength one ${ }^{4}$ and the Greek indices run over $n$ values $(n \geq 3)$. The gauge parameter $\varepsilon$ is a completely symmetric tensor field of rank $s-1$. For spin $s=2$, the gauge field $\varphi_{\mu \nu}$ represents the graviton while the gauge transformations (1) correspond to linearized diffeomorphisms.

Originally, some algebraic constraints were imposed on the metric-like gauge field and gauge parameters $[1,3]$. More precisely, given $\eta_{\mu \nu}$ the metric tensor of the flat background ${ }^{5}$,

\footnotetext{
${ }^{3}$ Throughout this text, the spin $s$ is taken to be a strictly positive integer. Note that the half-integer spin case is also covered here since all the results apply to fermions by simply replacing the spin $s$ by its integer part $[s]$.

${ }^{4}$ For example, $\varphi_{\left(\mu_{1} \ldots \mu_{s}\right)} \equiv \varphi_{\mu_{1} \ldots \mu_{s}}$.

${ }^{5}$ Our results are independent of the choice of signature because they rely on algebraic considerations only. For physical reasons, we choose the Lorentzian signature.
} 
the gauge parameter $\varepsilon$ was taken to be "traceless" $(s \geq 3)$ and the gauge field $\varphi$ to be "double-traceless" $(s \geq 4)$.

Notation: The trace of any tensor $T_{\mu_{1} \ldots \mu_{r}}$ is written as $T_{\mu_{1} \ldots \mu_{r-2}}^{\prime}\left(\equiv \eta^{\nu_{1} \nu_{2}} T_{\mu_{1} \ldots \mu_{r-2} \nu_{1} \nu_{2}}\right)$. Thus the previous "trace" constraints are written as

$$
\varepsilon_{\mu_{1} \ldots \mu_{s-3}}^{\prime}=0, \quad \varphi_{\mu_{1} \ldots \mu_{s-4}}^{\prime \prime}=0 .
$$

The traceless part of any tensor $T_{\mu_{1} \ldots \mu_{r}}$ is denoted by $\widehat{T}_{\mu_{1} \ldots \mu_{r}}$ (that is $\left.\widehat{T}_{\mu_{1} \ldots \mu_{r-2}}^{\prime}=0\right)$. Analogously, the "double traceless" part of $T$ is denoted by $\widetilde{T}_{\mu_{1} \ldots \mu_{r}}\left(\widetilde{T}_{\mu_{1} \ldots \mu_{r-4}}^{\prime \prime}=0\right)$. Hence, the constraints (2) can also be written

$$
\varepsilon_{\mu_{1} \ldots \mu_{s-1}}=\widehat{\varepsilon}_{\mu_{1} \ldots \mu_{s-1}}, \quad \varphi_{\mu_{1} \ldots \mu_{s}}=\widetilde{\varphi}_{\mu_{1} \ldots \mu_{s}} .
$$

Recently [5], Francia and Sagnotti realized that the algebraic constraints (2) could be consistently relaxed in the sense that the former theory appears as a gauge fixing of a consistent non-local theory without any trace constraint. The former and latter formulations will be referred to as "constrained" and "unconstrained" approaches (see [6] for pedagogical reviews on both of them). Though the absence of the constraints (2) simplifies our algebraic analysis, the non-locality property of the unconstrained approach is still rather elusive, therefore both cases will be treated for the sake of completeness.

\subsection{Gauge invariant functions}

There is a general belief that a local function of the completely symmetric gauge field $\varphi_{\mu_{1} \ldots \mu_{s}}$ is gauge invariant under unconstrained gauge transformations if and only if it depends on $\varphi$ only via the de Wit-Freedman curvature tensor field [3]

$$
\mathcal{R}_{\mu_{1} \ldots \mu_{s}, \nu_{1} \ldots \nu_{s}}:=\partial_{\mu_{1}} \ldots \partial_{\mu_{s}} \varphi_{\nu_{1} \ldots \nu_{s}}+\ldots
$$

and its partial derivatives. The dots stand for the terms necessary for the tensor $\mathcal{R}$ to belong to the irreducible $\mathfrak{g l}(n)$-module associated with the rectangular two-row Young diagram of length $s$ depicted ${ }_{s}^{s}$, so that $\mathcal{R}$ satisfies the algebraic identities

$$
\begin{gathered}
\mathcal{R}_{\mu_{1} \ldots \mu_{s}, \nu_{1} \ldots \nu_{s}}=\mathcal{R}_{\left(\mu_{1} \ldots \mu_{s}\right), \nu_{1} \ldots \nu_{s}}=\mathcal{R}_{\mu_{1} \ldots \mu_{s},\left(\nu_{1} \ldots \nu_{s}\right)}, \\
\mathcal{R}_{\left(\mu_{1} \ldots \mu_{s}, \nu_{1}\right) \nu_{2} \ldots \nu_{s}}=0 .
\end{gathered}
$$

For constrained fields and parameters, the most general local gauge invariant function of $\varphi$ is believed to depend only on the de Wit-Freedman curvature tensor $\mathcal{R}$ and on the Fronsdal tensor

$$
\mathcal{F}_{\mu_{1} \ldots \mu_{s}}:=\square \varphi_{\mu_{1} \ldots \mu_{s}}-s \partial^{\nu} \partial_{\left(\mu_{1}\right.} \varphi_{\left.\mu_{2} \ldots \mu_{s}\right) \nu}+\frac{s(s-1)}{2} \partial_{\left(\mu_{1}\right.} \partial_{\mu_{2}} \varphi_{\left.\mu_{3} \ldots \mu_{s}\right) \nu_{1} \nu_{2}}^{\prime}
$$

together with their derivatives.

We would like to put the previous expectations on firm mathematical grounds. Part of the present paper (Section 4.1) is therefore mainly devoted to a mathematically rigorous analysis of the following problem: the determination of all possible gauge invariant local functions depending on the gauge field $\varphi$ and a finite number of its partial derivatives both 
in the unconstrained and constrained approaches. Actually, the answer depends on whether the system is studied on-shell or not since, for instance,

$$
\mathcal{F}_{\mu_{1} \ldots \mu_{s}} \approx 0
$$

in the constrained approach. The symbol $\approx$ means that the equality holds on-shell. By combining the Damour-Deser identity [7] (which relates the trace of $\mathcal{R}$ to $s-2$ exterior derivatives of $\mathcal{F}$ ) with the generalized Poincaré lemma [8], we argued in [9] that the field equation

$$
\mathcal{R}_{\mu_{1} \ldots \mu_{s}, \nu_{1} \ldots \nu_{s-2}}^{\prime} \approx 0
$$

in the unconstrained approach is dynamically equivalent to (5) in the constrained approach. More precisely,

$$
\begin{aligned}
\mathcal{F}_{\mu_{1} \ldots \mu_{s}}(\widetilde{\varphi})= & 0, \delta \widetilde{\varphi}_{\mu_{1} \ldots \mu_{s}}=s \partial_{\left(\mu_{1}\right.} \widehat{\varepsilon}_{\left.\mu_{2} \ldots \mu_{s}\right)} \\
& \Longleftrightarrow \mathcal{R}_{\mu_{1} \ldots \mu_{s}, \nu_{1} \ldots \nu_{s-2}}^{\prime}(\varphi)=0, \quad \delta \varphi_{\mu_{1} \ldots \mu_{s}}=s \partial_{\left(\mu_{1}\right.} \varepsilon_{\left.\mu_{2} \ldots \mu_{s}\right)} .
\end{aligned}
$$

The spin- $s$ Weyl-like tensor $\mathcal{W}$ is defined as the traceless part of the spin- $s$ tensor $\mathcal{R}$,

$$
\mathcal{W}_{\mu_{1} \ldots \mu_{s}, \nu_{1} \ldots \nu_{s}}:=\widehat{\mathcal{R}}_{\mu_{1} \ldots \mu_{s}, \nu_{1} \ldots \nu_{s}}
$$

hence it belongs to the irreducible $\mathfrak{o}(n-1,1)$-module associated with the rectangular two-row Young diagram of length $s$. In both approaches, the on-shell de Wit - Freedman tensor is equal to the Weyl-like tensor

$$
\mathcal{R}_{\mu_{1} \ldots \mu_{s}, \nu_{1} \ldots \nu_{s}} \approx \mathcal{W}_{\mu_{1} \ldots \mu_{s}, \nu_{1} \ldots \nu_{s}} .
$$

A prerequisite of Vasiliev's unfolded approach is the determination of all on-shell nontrivial derivatives of the fieldstrengths. The spin- $s$ de Wit - Freedman tensor obeys some differential Bianchi identity and, on-shell, it is traceless, divergenceless and satisfies the massless KleinGordon equation [9]. This implies that the $k$ th partial derivatives of the spin- $s$ de Wit Freedman tensor belong off(on)-shell to the irreducible $\mathfrak{g l}(n)(\mathfrak{o}(n-1,1))$-module labeled by the two-row Young diagram $\longleftarrow s+k$. They span the so-called "twisted adjoint module" and play a fundamental role in the unfolded approach.

To address the previously-stated problem of determining the gauge-invariant local functions of the (un)constrained symmetric gauge field $\varphi$, we reformulate it as a cohomological problem in the BRST formalism (see e.g. [10] for a comprehensive review). More precisely, we use the fact that the set of such gauge invariant functions is given by the local BRST cohomology group $H^{0}(s)$ in vanishing ghost number in the sector without antifields. This cohomology group is actually identical to the cohomology group $H^{0}(\gamma)$ of the longitudinal exterior differential $\gamma$ in the sector without antifields. In the present paper, the complete local cohomology of $\gamma$ is determined. This result is useful because the knowledge of $H^{*}(\gamma)$ is the first ingredient in the computation of the local BRST cohomology group $H^{n, 0}(s \mid d)$ in top form degree and in vanishing ghost number for higher-spin gauge theories, a subject which will be addressed later in the context of the classification of local consistent vertices for higher-spin gauge theories in flat space-time [11].

These results will be compared to two other cohomological analyses of somewhat related problems: the generalized Poincaré lemma of [8] (extended to arbitrary irreducible tensors under the general linear group in [12]) and the problem of writing down non-trivial equations in the unfolded formalism [13] (see also Sections 9 and 10 of [2] for a review). 


\section{$2.2 \quad$ Killing tensors}

Another problem of physical interest for a better understanding of the higher-spin symmetries is the determination of all Killing tensor fields on Minkowski space-time, that is, the symmetric tensor fields satisfying the following (off-shell) Killing-like equation [14]

$$
\partial_{\left(\mu_{1}\right.} \varepsilon_{\left.\mu_{2} \ldots \mu_{s}\right)}=0 .
$$

Actually this problem is easy to solve for tensor fields $\varepsilon_{\mu_{1} \ldots \mu_{s-1}}(x)$ which are formal power series in $x$. Killing tensor fields on spaces of constant curvature have been extensively studied by mathematicians (see e.g. $[15,16]$ ).

A more involved problem is the determination of all local "on-shell Killing tensor fields". Non-trivial on-shell Killing tensor fields on flat space-time are solutions of the conditions

$$
\partial_{\left(\mu_{1}\right.} \varepsilon_{\left.\mu_{2} \ldots \mu_{s}\right)} \approx 0, \quad \varepsilon_{\mu_{1} \ldots \mu_{s-1}} \not \approx 0,
$$

which define a cohomological problem. Again, in order to address this problem we formulate it in the context of the local BRST formalism, by using the fact that these local non-trivial on-shell Killing tensor fields are in one-to-one correspondence with cocycles of the local Koszul-Tate cohomology group $H_{2}^{n}(\delta \mid d)$ in top form degree and antifield number two [17]. Though interesting in its own sake, the knowledge of $H_{2}^{n}(\delta \mid d)$ is the second ingredient in the computation of the local BRST cohomology group $H^{n, 0}(s \mid d)[11]$.

Generally speaking, the global symmetries of a solution of some field equation correspond to the space of gauge parameters leaving the gauge fields invariant under gauge transformations evaluated at the solution. Furthermore, for the flat vacuum solution they are expected to correspond to the full symmetry algebra of the theory. More specifically, the Killing tensors (8) of the infinite tower of higher-spin fields should be related to a higher-spin algebra (if any) in flat space-time. Indeed, we prove in Corollary 3 (Subsection 4.2) that they are in one-to-one correspondence with the elements of a Minkowski higher-spin algebra that can be obtained as a quotient of the universal enveloping algebra $\mathcal{U}(\mathfrak{i s o}(n-1,1))$ of the Poincaré algebra or as an Inönü-Wigner contraction of the anti de Sitter / conformal higher-spin algebras of Eastwood and Vasiliev [18, 19] in the flat limit $\Lambda \rightarrow 0$.

These higher-spin gauge symmetry algebras might eventually find their origin in the general procedure of "gauging" some global higher-symmetry algebras of free theories, as we argue now. Reformulating an observation of [20], the action of a complex bosonic field $\phi_{\mu_{1} \ldots \mu_{t}}$ with arbitrary spin $t$ is invariant under the global infinitesimal transformations

$$
\delta_{\lambda} \phi_{\mu_{1} \ldots \mu_{t}}=i^{s-2} \lambda_{\nu_{1} \ldots \nu_{s-1}} \partial^{\nu_{1}} \ldots \partial^{\nu_{s-1}} \phi_{\mu_{1} \ldots \mu_{t}},
$$

where $\lambda_{\nu_{1} \ldots \nu_{s-1}}$ are completely symmetric constant real tensors. For $s=1$, one recovers the usual infinitesimal $\mathfrak{u}(1)$ phase transformation and, for $s=2$, one obtains the usual infinitesimal action of the translation group $\mathbb{R}^{d}$. If one tried to gauge the global symmetry transformations of the form (10) by replacing the constant parameters $\lambda_{\nu_{1} \ldots \nu_{s-1}}$ by arbitrary completely symmetric tensor fields $\varepsilon_{\nu_{1} \ldots \nu_{s-1}}$, then the usual prescription would require the introduction of some connection in order to define proper covariant derivative. It is suggestive to interpret the gauge fields $\varphi_{\mu_{1} \ldots \mu_{s}}$ as entering into the definition of the connection so that the Abelian gauge transformations (1) should somehow correspond to the linearization of the non-Abelian gauge transformations. Moreover, the trace condition on the gauge parameter 
may find a natural explanation according to this line of thinking. For a scalar field $(t=0)$, the infinitesmal transformation (10) reads

$$
\delta_{\lambda} \phi \approx i^{s-2} \widehat{\lambda}_{\nu_{1} \ldots \nu_{s-1}} \partial^{\nu_{1}} \ldots \partial^{\nu_{s-1}} \phi
$$

that is, the parameters may be assumed to be traceless without loss of generality, since $\left(\square-m^{2}\right) \phi \approx 0$. This is in agreement with the fact that trace conditions are present in the so-called "on-shell" higher-spin algebra and not in the "off-shell" one (see Section 5 of [2]).

Let $\left\{P^{\mu}\right\}$ be a basis of the (Abelian) translation algebra $\mathbb{R}^{n}$ that is represented by the Hermitian operators $\mathrm{P}^{\mu}=i \partial^{\mu}$ acting on $\phi_{\mu_{1} \ldots \mu_{t}}$. The Hermitian kinetic operator $\mathrm{K}(\mathrm{P})$ for the field $\phi_{\mu_{1} \ldots \mu_{t}}$ is an element of the polynomial algebra $\mathbb{R}\left[\mathrm{P}^{\mu}\right]$. The translation operators $-i \lambda_{\mu} \mathrm{P}^{\mu}$ obviously generate symmetries of the quadratic action $\int\langle\phi|\mathrm{K}| \phi\rangle$ since they are antiHermitian and commute with the kinetic operator, but the same is true for any product of such translation operators, therefore the universal enveloping algebra of the translations generates an infinite-dimensional algebra of global symmetries of the free theory. The universal enveloping algebra of an Abelian Lie algebra is the corresponding polynomial algebra, in our case $\mathcal{U}\left(\mathbb{R}^{n}\right) \cong \mathbb{R}\left[P^{\mu}\right]$. This algebra is unitarily represented by the anti-Hermitian operators $-i \lambda_{\nu_{1} \ldots \nu_{s-1}} \mathrm{P}^{\nu_{1}} \ldots \mathrm{P}^{\nu_{s-1}}$ acting on the field $\phi_{\mu_{1} \ldots \mu_{t}}$ as in (10). (The symmetry algebra $\mathbb{R}\left[\mathrm{P}^{\mu}\right]$ should be quotiented by the ideal of elements proportional to $K(P)$ in order to obtain the algebra of non-trivial symmetries of the action. For $t=0$ and $\mathrm{K}=\mathrm{P}^{2}+m^{2}$, the action of the latter algebra on the scalar $\phi$ is written explicitly in (11).) The Abelian Lie algebra $^{6} \mathrm{hs}\left(\mathbb{R}^{n}\right)$ of the higher-symmetries (10) is obtained from the associative algebra $\mathcal{U}\left(\mathbb{R}^{n}\right)$ with the Lie bracket given by the commutator. The symmetry group $\operatorname{HS}\left(\mathbb{R}^{n}\right)$ is obtained by exponentiation of $\mathrm{hs}\left(\mathbb{R}^{n}\right)$ with generic element $U$ in the unitary representation:

$$
U=\exp \left(-i \lambda_{\nu_{1} \ldots \nu_{s-1}} \mathrm{P}^{\nu_{1}} \ldots \mathrm{P}^{\nu_{s-1}}\right) \text {. }
$$

This leads to the finite form

$$
\langle x \mid \phi\rangle \longrightarrow\langle x|U| \phi\rangle=\int d^{n} y U(x-y)\langle y \mid \phi\rangle=\exp (-i \lambda)\langle x \mid \phi\rangle+\langle x+\lambda \mid \phi\rangle+\ldots
$$

of the infinitesimal transformations (10) where

$$
U(z)=(2 \pi)^{-n} \int d^{n} p \exp \left[-i\left(z^{\mu} p_{\mu}+\lambda_{\nu_{1} \ldots \nu_{s-1}} p^{\nu_{1}} \ldots p^{\nu_{s-1}}\right)\right]
$$

and the dots in (12) denote the transformations corresponding to $s \geq 3$. These new terms are readily seen to be non-local (in the sense that they cannot be written in the form $\left\langle x^{\prime} \mid \phi\right\rangle$ where $\left.x^{\prime}=f(x)\right)$ because $U(z)$ is a delta of Dirac only when $s=1,2$.

The previous reasoning entirely applies to the more complicated Poincaré algebra iso $(n-$ 1,1 ) case and leads to some Minkowski higher-spin algebra. One should point out that the previous arguments provide a mean to evade the conclusions of the S-matrix no-go theorems against higher space-time symmetries [21], even in flat space-time. (For a more general discussion of higher-symmetries along the lines of this subsection, see [22].)

\footnotetext{
${ }^{6}$ hs stands at the same time for "higher-spin" and "higher-symmetry".
} 


\section{Cohomological reformulation of the problems}

\subsection{Jet space}

To reformulate a field theoretical problem - a functional problem - into a finite-dimensional algebraic problem - much more easy to cope with - one usually treats the fields and their partial derivatives as independent coordinates of a so-called "jet space". (We follow closely the terminology of the report [17].)

Notation: Frequently, we will omit indices for fields whose rank has been defined previously. The letter $\Phi$ will collectively denote the set of field variables (some of which may be Grassmannian). The $p$ th partial derivatives of the variables $\Phi$ will be denoted by $\partial^{p} \Phi$, that is $\partial^{p} \Phi \sim \partial_{\mu_{1}} \ldots \partial_{\mu_{p}} \Phi$.

An (off-shell) local function of the field variables $\Phi$ is a function $f=f(x,[\Phi])$ of the spacetime coordinates, polynomial in the field variables $\Phi$ and a finite number of their derivatives. The notation $[\Phi]$ means dependence on the variables $\Phi, \partial \Phi, \partial^{2} \Phi, \ldots, \partial^{k} \Phi$ for some finite but otherwise arbitrary integer $k$. The jet space of order $k$ is defined as the direct product $J^{k}(E)=\mathcal{M} \times V^{k}$, where $\mathcal{M}$ is the $n$-dimensional space-time manifold, $V^{k}$ the space with coordinates given by $\Phi, \partial \Phi, \partial^{2} \Phi, \ldots, \partial^{k} \Phi$ and $E \equiv J^{0}(E)$ the jet space of order zero with coordinates $x$ and $\Phi$. A local function is thus a function on a jet space of some finite order (which will always be omitted in the sequel for the sake of readability), that is, an element of the space of sections on the trivial bundle $J^{*}(E)$. We will denote the supercommutative algebra of (off-shell) local functions of the field variables $\Phi$ by $\Upsilon_{0}(\Phi)$.

Let $\Psi \in \Upsilon_{0}(\Phi)$ be the left-hand-side of the equations of motion $\Psi([\Phi]) \approx 0$. The collection of equations $[\Psi]=0$ defines a submanifold of the jet space $J^{*}(E)$ called the stationary surface and denoted by $\Sigma$. It turns out to be convenient to impose the following regularity conditions: (i) the local functions $[\Psi]$ can be split into independent and dependent ones and (ii) the independent functions can be locally taken as the first coordinates of a new, regular, coordinate system on the jet space $J^{*}(E)$ in the vicinity of the stationary surface $\Sigma$. The algebra of local functions proportional to the variables $[\Psi]$ forms an ideal, which we denote by $\mathcal{I}$, that leads to the equivalence relation

$$
f, g \in \Upsilon_{0}(\Phi) ; \quad f \approx g \Longleftrightarrow f-g \in \mathcal{I}
$$

One defines the algebra $\Upsilon_{\Psi}(\Phi)$ of on-shell local functions as the quotient of the algebra $\Upsilon_{0}(\Phi)$ by the ideal $\mathcal{I}$. The regularity conditions imply that the space of on-shell local functions is isomorphic to the space $\Gamma(\Sigma)$ of local functions on the stationary surface $\Sigma$.

We will not consider topologically non-trivial space-time and/or field manifolds. Therefore a field history is defined as a map $h: \mathcal{M} \rightarrow E: x \mapsto(x, \Phi(x))$. (In the more general case, one should now introduce sections instead of functions, as well as jet bundles.) Any field history naturally induces a map from $\mathcal{M}$ to any given jet space $J^{k}(E)$ by evaluating the partial derivatives at each point of $\mathcal{M}$. The evaluation of a local function at a field history yields a local space-time function. 


\subsection{Fields and antifields}

It is straightforward to apply the antifield BRST formalism ${ }^{7}$ to free "irreducible" gauge theories, such as Fronsdal's theory describing rank- $s$ completely symmetric gauge fields. Therefore we directly give the main results.

Let us start with the unconstrained gauge theory. The gauge transformations (1) are irreducible, hence it is sufficient to introduce a fermionic ghost field $\xi_{\mu_{1} \ldots \mu_{s-1}}$ for each bosonic gauge parameter $\varepsilon_{\mu_{1} \ldots \mu_{s-1}}$. The "field" content is thereby enlarged to

$$
\Phi=\{\varphi, \xi\}, \quad \epsilon(\varphi)=0, \quad \epsilon(\xi)=1,
$$

where $\epsilon(Z)$ denotes the Grassmann parity of the field $Z$. The corresponding algebra $\Upsilon_{0}(\Phi)$ of local functions of the fields is $\mathbb{N}$-graded by the pureghost number. The corresponding diagonal operator puregh is an even derivation defined by the following grading of the generators

$$
\operatorname{puregh}(\varphi)=0, \quad \operatorname{puregh}(\xi)=1 .
$$

To each field $Z \in \Phi$ we associate an antifield $Z^{*}$ of opposite parity. The set of associated antifields is then

$$
\Phi^{*}=\left\{\varphi^{*}, \xi^{*}\right\}, \quad \epsilon\left(\varphi^{*}\right)=1, \quad \epsilon\left(\xi^{*}\right)=0 .
$$

The pure ghost number of any antifield vanishes. The algebra $\Upsilon\left(\Phi, \Phi^{*}\right)$ of local functions of the fields and antifields is bigraded: first by the pureghost number, second by the antighost number defined from

$$
\operatorname{antigh}(\varphi)=0, \quad \operatorname{antigh}(\xi)=0 \quad \operatorname{antigh}\left(\varphi^{*}\right)=1, \quad \operatorname{antigh}\left(\xi^{*}\right)=2
$$

The (total) ghost number equals the difference between the pure ghost number and the antighost number

$$
g h(Z)=\operatorname{puregh}(Z)-\operatorname{antigh}(Z), \quad \forall Z \in \Phi \cup \Phi^{*} .
$$

The previous paragraph similarly applies to the constrained gauge theory, the only difference being that some trace constraints must be imposed. The field content of the constrained theory is

$$
\bar{\Phi}=\{\widetilde{\varphi}, \widehat{\xi}\}, \quad \epsilon(\widetilde{\varphi})=0, \quad \epsilon(\widehat{\xi})=1 .
$$

The set of associated antifields is then

$$
\bar{\Phi}^{*}=\left\{\widetilde{\varphi}^{*}, \widehat{\xi}^{*}\right\}, \quad \epsilon\left(\widetilde{\varphi}^{*}\right)=1, \quad \epsilon\left(\widehat{\xi}^{*}\right)=0 .
$$

Following the comments made in the introduction, the stationary surface is the submanifold defined by $\mathcal{R}^{\prime}([\varphi]) \approx 0$ in the unconstrained approach and by $\mathcal{F}([\widetilde{\varphi}]) \approx 0$ in the constrained approach. Thus the algebras of on-shell local functions are respectively $\Upsilon_{\mathcal{R}^{\prime}}(\varphi)$ in the unconstrained approach and $\Upsilon_{\mathcal{F}}(\widetilde{\varphi})$ in the constrained approach.

\footnotetext{
${ }^{7}$ For reviews on the Lagrangian (i.e. antifield) BRST method, we refer the reader to the book [10] and the lectures [23].
} 


\subsection{BRST differential}

The BRST differential $s$ acts on the algebra $\Upsilon_{0}\left(\Phi, \Phi^{*}\right)$ and its ghost number is equal to one. Its action obviously induces an action on the subalgebra $\Upsilon_{0}\left(\bar{\Phi}, \bar{\Phi}^{*}\right)$. It decomposes according to the antighost number as

$$
s=\delta+\gamma, \quad \operatorname{antigh}(\delta)=-1, \quad \operatorname{antigh}(\gamma)=0
$$

and provides the algebra of gauge invariant functions on the stationary surface through its cohomology at ghost number zero $H^{0}(s)$. The Koszul-Tate differential $\delta$ acts trivially on the fields, $\delta \Phi=0$. In the antifield sector, $\delta \varphi^{*}$ is equal to the equations of motion and $\delta \xi^{*}$ is proportional to the "Noether identities". The exterior derivative along the gauge orbits is the differential $\gamma$ defined by replacing the gauge parameters by the corresponding ghosts in the gauge transformation (1)

$$
\gamma \varphi_{\mu_{1} \ldots \mu_{s}}=s \partial_{\left(\mu_{1}\right.} \xi_{\left.\mu_{2} \ldots \mu_{s}\right)}, \quad \gamma \xi_{\mu_{1} \ldots \mu_{s-1}}=0
$$

On the one hand, the Koszul-Tate differential $\delta$ implements the restriction on the stationary surface $\Sigma$ (since it provides a resolution of the algebra of functions on the stationary surface). On the other hand, the longitudinal exterior differential $\gamma$ picks out the gauge-invariant functions via its cohomology at pureghost number zero. The cohomological groups $H^{0}\left(s, \Upsilon_{0}\left(\Phi, \Phi^{*}\right)\right) \cong H\left(\gamma, \Upsilon_{\mathcal{R}^{\prime}}(\varphi)\right)$ and $H^{0}\left(s, \Upsilon_{0}\left(\bar{\Phi}, \bar{\Phi}^{*}\right)\right) \cong H\left(\gamma, \Upsilon_{\mathcal{F}}(\widetilde{\varphi})\right)$ can be read off Corollary 1 given in the next section.

\section{The results and their physical interpretations}

\subsection{Longitudinal exterior cohomology}

Let us define the Grassmann algebra $\Xi$ generated by the fermionic variables $d^{k} \xi$, where $k$ is a positive integer not larger than $s-1$ and where each exterior derivative $d$ in the power $d^{k}$ acts on a different space-time index of $\xi$. It is easy to see that the tensors $d^{k} \xi$ belong to the irreducible $\mathfrak{g l}(n)$-module labeled by the Young diagram $\square \mathrm{k}-1$. The set of variables $\xi, d \xi, d^{2} \xi, \ldots, d^{s-1} \xi$ is collectively denoted by $\langle\xi\rangle$. The traceless parts of the set of constrained variables $\widehat{\xi}, d \widehat{\xi}, d^{2} \widehat{\xi}, \ldots, d^{s-1} \widehat{\xi}$ is collectively denoted by $\{\widehat{\xi}\}$ and the corresponding Grassmann algebra by $\widehat{\Xi}$.

Theorem 1. For the unconstrained gauge theory, the cohomology of the longitudinal exterior differential $\gamma$ is the superalgebra freely generated by

- the space-time coordinates $x^{\mu}$,

- the curvature tensor $\mathcal{R}$ with its partial derivatives,

- the ghost $\xi$ and its $s-1$ exterior derivatives,

- the antifields $\Phi^{*}$ with their partial derivatives, 
that is,

$$
H\left(\gamma, \Upsilon_{0}\left(\Phi, \Phi^{*}\right)\right) \cong \Upsilon_{0}\left(\mathcal{R}, \Phi^{*}\right) \otimes \Xi
$$

More explicitly,

$$
\begin{gathered}
\gamma F\left(x,[\Phi],\left[\Phi^{*}\right]\right)=0 \quad \Longleftrightarrow \quad F=G\left(x,[\mathcal{R}],\langle\xi\rangle,\left[\Phi^{*}\right]\right)+\gamma(\ldots), \\
G\left(x,[\mathcal{R}],\langle\xi\rangle,\left[\Phi^{*}\right]\right)=\gamma(\ldots) \quad \Longleftrightarrow \quad G=0 .
\end{gathered}
$$

For the constrained gauge theory, the cohomology of the longitudinal exterior differential $\gamma$ is the superalgebra freely generated by

- the space-time coordinates $x^{\mu}$,

- the traceless components $\widehat{[\mathcal{R}]}$ of (the partial derivatives of) the curvature tensor,

- the Fronsdal tensor $\mathcal{F}$ and its partial derivatives,

- the traceless components of the ghost $\widehat{\xi}$ and its $s-1$ exterior derivatives,

- the antifields $\bar{\Phi}^{*}$ with their partial derivatives,

that $i s^{8}$

$$
H\left(\gamma, \Upsilon_{0}\left(\bar{\Phi}, \bar{\Phi}^{*}\right)\right) \cong \Upsilon_{0}\left(\mathcal{R}, \mathcal{F}, \bar{\Phi}^{*}\right) \otimes \widehat{\Xi}
$$

More explicitly,

$$
\begin{gathered}
\gamma F\left(x,[\bar{\Phi}],\left[\bar{\Phi}^{*}\right]\right)=0 \quad \Longleftrightarrow \quad F=G\left(x, \widehat{[\mathcal{R}]},[\mathcal{F}],(\widehat{\xi}),\left[\bar{\Phi}^{*}\right]\right)+\gamma(\ldots), \\
G\left(x, \widehat{[\mathcal{R}]},[\mathcal{F}],\{\widehat{\xi}\},\left[\bar{\Phi}^{*}\right]\right)=\gamma(\ldots) \quad \Longleftrightarrow \quad G=0 .
\end{gathered}
$$

Setting the ghosts and antifields to zero, one can make contact with the standard physical field content. Moreover, by restricting the gauge-invariant functions to the stationary surfaces defined by the relations (5) or (6), one may even put them on-shell in the corresponding approaches. The results are summarized in the following corollary of Theorem 1:

Corollary 1. - A local function of the unconstrained gauge field $\varphi$ is gauge invariant under unconstrained gauge transformations if and only if it depends on $\varphi$ via the de Wit-Freedman curvature tensor field $\mathcal{R}$ and its partial derivatives. Therefore, on-shell it is a function of the traceless component of the partial derivatives of the Weyl-like tensor $\mathcal{W}$ only.

$$
\delta_{\varepsilon} f([\varphi])=0, \quad \forall \varepsilon \Longleftrightarrow f=f([\mathcal{R}]) \Longleftrightarrow f \approx f(\widehat{[\mathcal{W}]})
$$

- A local function of the unconstrained gauge field $\varphi$ that is invariant under constrained gauge transformations must depend on the Fronsdal tensor $\mathcal{F}$ with all its partial derivatives,

\footnotetext{
${ }^{8}$ In the notation $\Upsilon_{0}\left(\mathcal{R}, \mathcal{F}, \bar{\Phi}^{*}\right)$, there is some redundacy in the sense that the variables $[\mathcal{R}]$ and $[\mathcal{F}]$ are not entirely independent.
} 
the traceless component of the partial derivatives of $\mathcal{R}$ and on the double trace $\varphi^{\prime \prime}$ of the gauge field.

$$
\delta_{\widehat{\varepsilon}} f([\varphi])=0, \quad \forall \widehat{\varepsilon} \quad \Longleftrightarrow f=f\left(\widehat{[\mathcal{R}]},[\mathcal{F}],\left[\varphi^{\prime \prime}\right]\right) .
$$

- For constrained gauge fields and parameters, the most general local gauge-invariant function must depend on $\widetilde{\varphi}$ only through the Fronsdal tensor $\mathcal{F}$ with all its partial derivatives and the traceless component of the partial derivatives of $\mathcal{R}$. Therefore, on-shell it is a function of the traceless component of the partial derivatives of the Weyl-like tensor $\mathcal{W}$ solely.

$$
\delta_{\widehat{\varepsilon}} g([\widetilde{\varphi}])=0, \quad \forall \widehat{\varepsilon} \quad \Longleftrightarrow g=g(\widehat{[\mathcal{R}]},[\mathcal{F}]) \quad \Longleftrightarrow g \approx f(\widehat{[\mathcal{W}]})
$$

where $f(\widehat{[\mathcal{R}]}):=g(\widehat{[\mathcal{R}]},[\mathcal{F}]=0)$.

Some remarks are in order:

- As one can see, the space of on-shell gauge invariant local functions are identical in both approaches. This confirms the fact that both approaches are dynamically equivalent, as written in (7). Moreover, this is in complete agreement with Vasiliev's unfolded formulation where the variables $\widehat{[\mathcal{W}]}$ span the on-shell twisted adjoint module for the free theory of a rank- $s$ symmetric tensor gauge field in flat space-time (see Sections 8 and 12 of [2]).

- Corollary 1 may also be used when writing down local free (i.e. linear) field equations that are gauge-invariant and translation-invariant. Indeed, Corollary 1 implies that the most general possibility is an equation that fixes to zero a local function linear in $[\mathcal{R}]$ in the unconstrained case and linear in $[\mathcal{R}]$ and $[\mathcal{F}]$ in the constrained approach. These possibilities reproduce the "Weyl cohomology" and "Einstein cohomology" obtained through the very different method of [13] (see also Section 10 of [2]).

- Notice that these algebraic results also hold for local spacetime functions obtained by the evaluation of the local function $f$ at an arbitrary field history. Such a spacetime version of Corollary 1 is somehow the converse of deriving the gauge transformations (1) as the most general transformations leaving invariant the curvature tensor $\mathcal{R}(x)$ and, in the constrained approach, the Fronsdal operator $\mathcal{F}(x)$. (This may be done as a straigthforward application of the results contained in [8].)

\subsection{Local Koszul-Tate cohomology}

The space $\mathcal{K}$ of unconstrained (off-shell) Killing tensor fields on flat space-time is the vector space of all completely symmetric tensor fields $\varepsilon_{\mu_{1} \ldots \mu_{r}}(x)$ satisfying $(8)$. The space $\mathcal{K}=\bigoplus_{r=0}^{\infty} \mathcal{K}_{r}$ is $\mathbb{N}$-graded by the rank $r$ and can be endowed with a structure of $\mathbb{N}$-graded commutative algebra via the natural symmetric product

$$
\left(\varepsilon_{1} \vee \varepsilon_{2}\right)_{\mu_{1} \ldots \mu_{r_{1}+r_{2}}}:=\left(\varepsilon_{1}\right)_{\left(\mu_{1} \ldots \mu_{r_{1}}\right.}\left(\varepsilon_{2}\right)_{\left.\mu_{r_{1}+1 \ldots \mu_{r_{1}+r_{2}}}\right)} .
$$

One can explicitly check that the rank- $\left(r_{1}+r_{2}\right)$ symmetric tensor field $\varepsilon_{1} \vee \varepsilon_{2}$ obeys the off-shell Killing-like equation. This ensures that the product $\vee$ is internal in the associative algebra $\mathcal{K}$. 
Lemma 1. For an unconstrained free spin-s $(s \geq 1)$ gauge field theory, the most general off-shell Killing tensor field $\varepsilon_{\mu_{1} \ldots \mu_{s-1}}(x)$ that is a formal power series in $x$ is a polynomial of degree $s-1$ where the coefficient of the term of homogeneity degree $t$ is an irreducible tensor

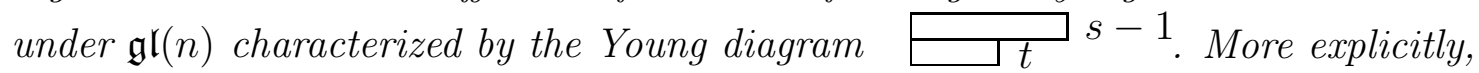

$$
\begin{gathered}
\partial_{\left(\mu_{1}\right.} \varepsilon_{\left.\mu_{2} \ldots \mu_{s}\right)}(x)=0 \\
\mathbb{\Downarrow} \\
\varepsilon_{\mu_{1} \ldots \mu_{s-1}}(x)=\sum_{t=0}^{s-1} \lambda_{\mu_{1} \ldots \mu_{s-1}, \nu_{1} \ldots \nu_{t}} x^{\nu_{1}} \ldots x^{\nu_{t}}, \quad \lambda_{\left(\mu_{1} \ldots \mu_{s-1}, \nu_{1}\right) \nu_{2} \ldots \nu_{t}}=0 .
\end{gathered}
$$

This lemma has been derived previously by several authors in different versions [16, 24]. In Appendix C.1, we provide a short and new proof of this useful property.

Our main results about Killing tensors (either off-shell or on-shell) are contained in the following theorem:

Theorem 2. (i) Let $\left\{P^{\mu}, M^{\nu \rho}\right\}$ be a basis of the Lie algebra $\mathfrak{i s o}(n-1,1):=\mathbb{R}^{n} \boxplus \mathfrak{s o}(n-$ $1,1)$ of the isometry group of the flat space-time $\mathbb{R}^{n-1,1}$, where $\left\{P^{\mu}\right\}$ is a basis of the translation algebra $\mathbb{R}^{n}$ and $\left\{M^{\mu \nu}\right\}$ is a basis of the Lorentz algebra $\mathfrak{s o}(n-1,1)$.

The commutative algebra $\mathcal{K}$ of unconstrained off-shell Killing tensor fields is isomorphic to the quotient of the symmetric algebra $\bigvee(\mathfrak{i s o}(n-1,1))$ of the vector space $\mathfrak{i s o}(n-1,1)$ by the relations

$$
R \equiv\left\{P_{[\mu} M_{\nu \rho]}, M_{[\mu \nu} M_{\rho] \sigma}\right\} .
$$

More precisely, any equivalence class of the algebra

$$
\mathcal{K} \cong \bigvee(\mathfrak{i s o}(n-1,1)) / R
$$

can be represented by a Weyl-ordered polynomial

$$
S(M, P)=\sum_{s \geq 1} \sum_{t=0}^{s-1} \zeta_{\mu_{1} \ldots \mu_{s-1}, \nu_{1} \ldots \nu_{t}}\left(M^{\nu_{1} \mu_{1}} \cdots M^{\nu_{t} \mu_{t}} P^{\mu_{t+1}} \cdots P^{\mu_{s-1}}+\text { perms }\right)
$$

where the coefficients $\zeta$ are tensors having the same symmetry properties as the tensors $\lambda$ in Lemma 1 and "perms" stands for the sum of terms obtained from $M^{\nu_{1} \mu_{1}} \cdots M^{\nu_{t} \mu_{t}}$ $P^{\mu_{t+1}} \cdots P^{\mu_{s-1}}$ by performing all nontrivial permutations of the elements $P^{\mu}$ and $M^{\mu \nu}$.

(ii) All non-trivial on-shell Killing tensor fields $\widehat{\varepsilon}_{\mu_{1} \ldots \mu_{s-1}}(x,[\varphi])$ (that are formal power series in $x$ ) of the constrained theory of spin-s free gauge field can be represented by the traceless component of the off-shell Killing tensor fields given in Lemma 1.

By looking at the details of Theorems 6.5 and 6.7 of [17] (and their proofs), it is straightforward to see that the following corollary is equivalent to the point (ii) of Theorem 2 :

Corollary 2. In the constrained approach, the top forms $\widehat{\varepsilon}_{\mu_{1} \ldots \mu_{s-1}}(x) \widehat{\xi}^{* \mu_{1} \ldots \mu_{s-1}} d^{n} x$, where $\widehat{\varepsilon}_{\mu_{1} \ldots \mu_{s-1}}(x)$ runs over all non-trivial on-shell Killing tensor fields, span the local Koszul-Tate cohomology $H_{2}^{n}\left(\delta \mid d, \Upsilon_{0}\left(\bar{\Phi}, \bar{\Phi}^{*}\right)\right)$ in top form degree $n$ and in antifield number 2 . 
Let $\mathbb{R}[\mathrm{X}, \mathrm{P}]$ be the real polynomial algebra in the variables $\mathrm{X}^{\mu}$ and $\mathrm{P}^{\nu}$. Let us introduce the antisymetric bilinears $\mathrm{M}^{\mu \nu}:=\mathrm{X}^{\mu} \mathrm{P}^{\nu}-\mathrm{X}^{\nu} \mathrm{P}^{\mu}$ that provide a realization of the relations (16). The representative (17) in the corresponding realization of $\bigvee(\mathfrak{i s o}(n-1,1)) / R$ in $\mathbb{R}[\mathrm{X}, \mathrm{P}]$ is equal to

$$
\Lambda(\mathrm{X}, \mathrm{P})=\sum_{s \geq 1} \sum_{t=0}^{s-1} \lambda_{\mu_{1} \ldots \mu_{s-1}, \nu_{1} \ldots \nu_{t}} \mathrm{X}^{\nu_{1}} \ldots \mathrm{X}^{\nu_{t}} \mathrm{P}^{\mu_{1}} \ldots \mathrm{P}^{\mu_{s-1}}
$$

where the tensor $\lambda_{\mu_{1} \ldots \mu_{s-1}, \nu_{1} \ldots \nu_{t}}$ is a linear function of the tensor $\zeta_{\mu_{1} \ldots \mu_{s-1}, \nu_{1} \ldots \nu_{t}}$. They satisfy the same symmetry properties as the tensors $\lambda$ in Lemma 1 . The isomorphism between the algebra $\mathcal{K}$ of unconstrained off-shell Killing tensor fields (15) and the quotient $\bigvee(\mathfrak{i s o}(n-1,1)) / R$ is obvious when the representatives of the quotient are realized as in (18). In that case, the symmetrized tensor product (14) in $\mathcal{K}$ is mapped to the pointwise product of polynomials in $\mathbb{R}[\mathrm{X}, \mathrm{P}]$ induced by the following embeddings

$$
\mathcal{K}_{s-1} \hookrightarrow \mathbb{R}[\mathrm{X}, \mathrm{P}]: \varepsilon_{\mu_{1} \ldots \mu_{s-1}}(x) \mapsto \varepsilon_{\mu_{1} \ldots \mu_{s-1}}(\mathrm{X}) \mathrm{P}^{\mu_{1}} \ldots \mathrm{P}^{\mu_{s-1}} .
$$

The Poincaré-Birkhoff-Witt theorem shows that there exists a canonical isomorphism of vector spaces between the universal enveloping algebra $\mathcal{U}(\mathfrak{g})$ and the symmetric algebra $\bigvee(\mathfrak{g})$. Given a unitary representation of the Poincaré group $I S O(n-1,1)$ such that the generators $\mathrm{P}^{\mu}$ and $\mathrm{M}^{\nu \rho}$ are Hermitian operators acting on some Hilbert space $\{|\phi\rangle\}$, the corresponding Hermitian operators of the form (17) define a unitary representation of the universal enveloping algebra $\mathcal{U}(\mathfrak{i s o}(n-1,1))$. By definition, any Lorentz scalar $\mathrm{K}\left(\mathrm{P}^{2}\right)$ built out of the quadratic Casimir operator $\mathrm{P}^{2}$ commute with the generators $\mathrm{P}^{\mu}$ and $\mathrm{M}^{\nu \rho}$ of $\mathfrak{i s o}(n-1,1)$. Therefore, the Hermitian operators of the form (17) generate global symmetries of the quadratic action $\langle\phi|\mathrm{K}| \phi\rangle$ with kinetic operator $\mathrm{K}:=\mathrm{K}\left(\mathrm{P}^{2}\right)$.

The Weyl algebra $A_{2 n}$ is the quotient of the complex polynomial algebra $\mathbb{C}[\mathrm{X}, \mathrm{P}]$ by the commutation relations

$$
\mathrm{P}^{\mu} \mathrm{X}^{\nu}-\mathrm{X}^{\nu} \mathrm{P}^{\mu}=i \eta^{\mu \nu}
$$

A basis of the Lie algebra iso $(n-1,1)$ can be realized by the set $\left\{\mathrm{P}^{\mu}, \mathrm{M}^{\nu \rho}\right\} \subset A_{2 n}$. The "off-shell" Minkowski higher-spin Lie algebra $\mathrm{hs}_{\infty}(\mathfrak{i s o}(n-1,1))$ is defined by endowing the realization of $\mathcal{U}(\mathfrak{i s o}(n-1,1))$ in $A_{2 n}$ with a Lie algebra structure by means of the commutator.

If one considers only traceless tensors $\widehat{\zeta}$ in the expression (17) for the representatives of $\bigvee(\mathfrak{i s o}(n-1,1)) / R$, then, by making use of the commutation relation (20), the corresponding representatives $S(\mathrm{M}, \mathrm{P})$ can be rewritten as the normal-ordered polynomial

$$
H(\mathrm{X}, \mathrm{P}):=\sum_{s \geq 1} \sum_{t=0}^{s-1} \widehat{\lambda}_{\mu_{1} \ldots \mu_{s-1}, \nu_{1} \ldots \nu_{t}} \mathrm{X}^{\nu_{1}} \ldots \mathrm{X}^{\nu_{t}} \mathrm{P}^{\mu_{1}} \ldots \mathrm{P}^{\mu_{s-1}}
$$

where the constant tensors $\widehat{\lambda}_{\mu_{1} \ldots \mu_{s-1}, \nu_{1} \ldots \nu_{t}}$ belong to the irreducible $\mathfrak{o}(n-1,1)$-module labeled by the Young diagram $\underbrace{}_{t} s-1$. The elements (21) of the Weyl algebra are of physical interest because they commute with any Lorentz scalar $\mathrm{K}\left(\mathrm{P}^{2}\right)$ built out of $\mathrm{P}^{2}$.

Given a unitary representation of the Weyl algebra $A_{2 n}$ defined by (20) such that $\mathrm{X}^{\mu}$ and $\mathrm{P}^{\nu}$ are Hermitian, the Hermitian representatives (21) generate global symmetries of the quadratic action $\langle\phi|\mathrm{K}| \phi\rangle$ with kinetic operator $\mathrm{K}=\mathrm{K}\left(\mathrm{P}^{2}\right)$. For instance, the Hermitian operators $\mathrm{X}^{\mu}:=x^{\mu}$ and $\mathrm{P}^{\mu}:=i \partial^{\mu}$ define a unitary representation of the Weyl algebra $A_{2 n}$ 
on the functional space of square-integrable complex bosonic scalar fields $\phi$. The realization of the enveloping algebra $\mathcal{U}(\mathfrak{i s o}(n-1,1))$ in $A_{2 n}$ quotiented by the traces admits a faithful unitary representation acting as the global infinitesimal transformations

$$
\delta_{\widehat{\varepsilon}} \phi=i^{s-2} \widehat{\varepsilon}_{\nu_{1} \ldots \nu_{s-1}}(x) \partial^{\nu_{1}} \ldots \partial^{\nu_{s-1}} \phi
$$

where $\widehat{\varepsilon}(x)$ is an arbitrary traceless off-shell Killing tensor field. The higher-order global symmetries (22) should naturally give rise via the Noether theorem to the list of higher-spin conserved currents explicitly constructed in [27]. It is clear that the infinitesimal transformations (22) are related to the mappings (19) between Killing tensor fields and polynomials in $\mathrm{X}$ and $\mathrm{P}$ in the constrained approach. As explained in Subsection 2.2, for a scalar field, the trace in the parameter correspond to global transformations leaving the scalar field invariant on-shell. Factoring out the traces of $\mathrm{hs}_{\infty}(\mathfrak{i s o}(n-1,1))$ leads to the "on-shell" Minkowski higher-spin Lie algebra denoted by hs $(\mathfrak{i s o}(n-1,1))$.

Theorem 2 implies that, as suggested in the introduction, the infinite tower of Killing tensors in flat space-time is related to some Minkowski higher-spin algebra:

Corollary 3. The elements of the off-shell higher-spin algebra $\mathrm{hs}_{\infty}(\mathbf{i s o}(n-1,1))$ are in oneto-one correspondence with the off-shell Killing tensor fields $\varepsilon_{\nu_{1} \ldots \nu_{s-1}}$ of the unconstrained approach, while the elements of the on-shell higher-spin algebra $\mathrm{hs}(\mathfrak{i s o}(n-1,1))$ are in oneto-one correspondence with the non-trivial on-shell Killing tensor fields $\widehat{\varepsilon}_{\nu_{1} \ldots \nu_{s-1}}$ of the constrained approach.

The bijection is more manifest in the frame-like formulation. The constructions of the previous higher-spin algebras (together with their relationship with Killing tensors) are the analogue of the case of bosonic anti de Sitter / conformal higher-spin algebras [25, 18, 19, 26] (see also Section 5 of [2]) except that the Lie algebra $\mathfrak{i s o}(n-1,1)$ must be replaced by $\mathfrak{s o}(n-1,2)$. The former Lie algebra can be obtained from the latter as an Inönü-Wigner contraction in the flat limit $\Lambda \rightarrow 0$, therefore this also holds for the corresponding higher-spin algebras. During the redaction of the present paper, it has been shown by M.A. Vasiliev [28] that the algebra $\mathbf{h s}_{\infty}(\mathfrak{i s o}(n-1,1))$ corresponds to the global symmetries of Minkowski vacuum solution of the off-shell unfolded equations for the bosonic higher-spin gauge field theory [19]. This result confirms the relevance of our analysis.

Note added: After having submitted the paper to arXiv, we received the work [29] where the on-shell Killing tensors and the characteristic cohomology groups in form degrees $\leq n-2$ are computed for the constrained spin-3 theory; and we have been informed that slightly more general versions of the point (ii) of Theorem 2 and of Corollary 2 have been obtained independently by G. Barnich and N. Bouatta [30].

\section{Acknowledgements}

We thank L. Gualtieri for early discussions on the spin-3 case. 


\section{A Irreducible representations}

Most of the textbook material reviewed in this section can be extracted from [34]. In conformity with the mathematical literature, we adopt the Euclidean signature convention.

\section{A.1 Young diagrams}

Partition of integers play a key role in labeling the irreducible representations (irreps) of the general linear and orthogonal groups. The partition of the positive integer $|\lambda|$ into $r$ integer parts $\lambda_{1}, \lambda_{2}, \ldots, \lambda_{r}$ with $\lambda_{1}+\lambda_{2}+\ldots+\lambda_{r}=|\lambda|$ and $\lambda_{1} \geq \lambda_{2} \geq \ldots \geq \lambda_{r}>0$ is denoted by $\lambda=\left(\lambda_{1}, \lambda_{2}, \ldots, \lambda_{r}\right)$. Each such partition $\lambda$ specifies a Young diagram consisting of $|\lambda|$ boxes arranged in $r$ left-adjusted rows, where the length of the $i$ th row is $\lambda_{i}(i=1,2, \ldots, r)$.

Let $Y$ be the Abelian group made of all formal finite sums of Young diagrams with integer coefficients. This group is $\mathbb{N}$-graded by the number $|\lambda|$ of boxes: $Y=\sum_{n \in \mathbb{N}} Y_{n}$. The famous "Littlewood-Richardson rule" defines a multiplication law which endows $Y$ with a structure of graded commutative ring. The product of two Young diagrams $\lambda$ and $\mu$ is defined as the bilinear mapping to

$$
\lambda \cdot \mu=\sum_{\nu} m_{\lambda \mu \mid \nu} \nu
$$

where the coefficients $m_{\lambda \mu \mid \nu}=m_{\mu \lambda \mid \nu}$ are the number of distinct labeling of the Young diagram $\nu$ obtained from the following procedure:

1. Label the Young diagram $\mu$ by writing the letter "a" in all boxes of its first row, the letter "b" in all boxes of its second row, the letter "c" in all boxes of its third row, etc.

2. Add the labeled boxes of the Young tableau $\mu$ to $\lambda$ in Latin alphabetic order, one letter at a time and in such a way that at every stage:

(i) The resulting diagram is a Young diagram,

(ii) No two identical letters appear in the same column,

(iii) Reading from right to left across each row in turn from top to bottom (like in Arabic), the number of a's read should always be $\geq$ the number of b's read $\geq$ the number of c's read, etc.

As one can see, $|\lambda \cdot \mu|=|\lambda|+|\mu|$. A related operation in $Y$ is the "division" of $\nu$ by $\mu$ defined as the bilinear mapping to

$$
\nu / \mu=\sum_{\lambda} m_{\lambda \mu \mid \nu} \lambda
$$

where the sum is over Young diagrams $\lambda$ such that the product $\lambda \cdot \mu$ contains the term $\nu$ (with coefficient $m_{\lambda \mu \mid \nu}$ ).

The following obvious lemma will be used many times to simplify the computation of the cohomology of the differential $\gamma$ along the gauge orbits:

Lemma 2. Let $m$ and $n$ be two strictly positive integers such that $m \geq n$.

The product of two rows of respective lengths $m$ and $n$ is the sum of the two-row Young diagram obtained by putting the shortest row on the bottom of the longest and the product of two rows of respective lengths $m+1$ and $n-1$. 


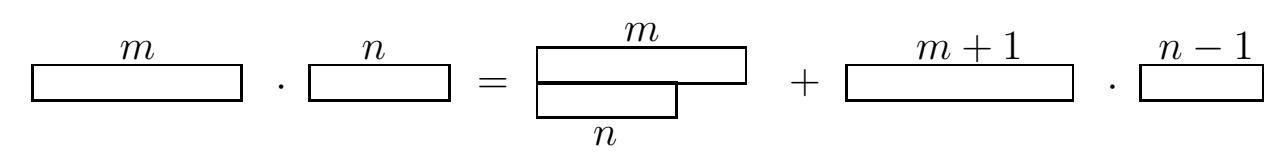

\section{A.2 Kronecker products and branching rules}

Irreps of $\mathfrak{g l}(n)$ may be labeled by $\{\lambda\}$ where the partition $\lambda$ serves to specify the symmetry properties of the corresponding rank- $|\lambda|$ covariant (or contravariant) tensors forming the basis of this irreducible Lie algebra module. Let $Y_{+}$be the Abelian monoid made of all formal finite sums of Young diagrams with non-negative integer coefficients. Finite direct sums of irreps of $\mathfrak{g l}(n)$ may therefore be labeled by $Y_{+}$via the rule

$$
\{m \mu+n \nu\}=m\{\mu\} \oplus n\{\nu\},
$$

where the positive integer coefficients $m, n \in \mathbb{N}$ must be interpreted as the multiplicity of the corresponding representation.

The evaluation of the Kronecker product of two $\mathfrak{g l}(n)$-irreps $\{\lambda\}$ and $\{\mu\}$ can be done by means of the Littlewood-Richardson rule which gives

$$
\{\lambda\} \otimes\{\mu\}=\{\lambda \cdot \mu\}=\bigoplus_{\nu} m_{\lambda \mu \mid \nu}\{\nu\} .
$$

A related operation is that of contraction of one set of contravariant indices of symmetry $\mu$ with a subset of a set of covariant tensor indices of symmetry $\nu$ to yield a sum of covariant tensors with indices of symmetry $\lambda$ given by the division rule

$$
\{\nu\} /\{\mu\}=\{\nu / \mu\}=\bigoplus_{\lambda} m_{\lambda \mu \mid \nu}\{\lambda\} .
$$

The irreps of $\mathfrak{g l}(n)$ may be reduced to irreps of $\mathfrak{o}(n)$ by extracting all possible trace terms formed by contraction with the metric tensor $\eta$ and its inverse. The corresponding irreps are labeled by $[\sigma]$. The reduction is given by the branching rule

$$
\mathfrak{g l}(n) \downarrow \mathfrak{o}(n) \quad: \quad\{\lambda\} \downarrow[\lambda / \Delta],
$$

where $\Delta$ is the formal infinite sum

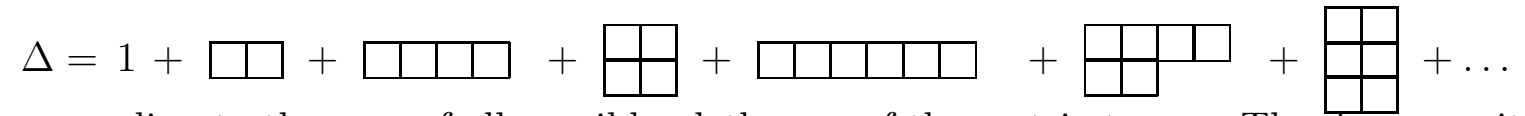

corresponding to the sum of all possible plethysms of the metric tensor. The decomposition (24) actually has a useful converse

$$
\mathfrak{o}(n) \uparrow \mathfrak{g l}(n) \quad: \quad[\lambda] \uparrow\left\{\lambda / \Delta^{-1}\right\},
$$

because the series $\Delta$ has an inverse

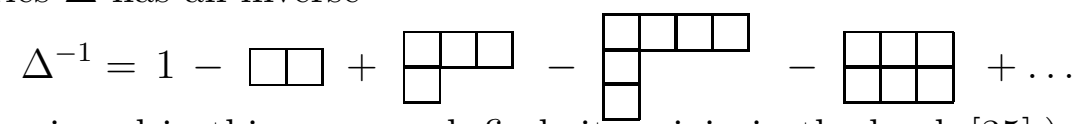

(The material reviewed in this paragraph finds its origin in the book [35].)

The operation (24) leads to a formal finite sum of irreps, some of which with strictly negative integer coefficients that have to be interpreted as constraints on some trace of the corresponding tensor basis. (Remark: These constraints are not preserved by the full $\mathfrak{g l}(n)$ algebra.) We introduce the notation

$$
\{\lambda-\mu\} \equiv\{\lambda\} \ominus\{\mu\},
$$

for later convenience. 


\section{B Proof of Theorem 1}

Our proof makes a decisive use of the simple fact that contractible pairs drop out from the (co)homology. The plan of our proof is therefore very simple: provide a new set of generators for which the contractible pairs are manifest. This convenient set of generators is identified via the decomposition of the jet bundle in irreducible modules of either $\mathfrak{g l}(n)$ or $\mathfrak{o}(n)$ algebras. This strategy follows the lines of previous computations of $H^{0}(\gamma)$ for other gauge theories, such as completely antisymmetric [31], spin-two [32] and two-column mixed symmetry [33] gauge fields.

\section{B.1 Contractible pairs}

Let $\mathcal{A}$ be the supercommutative differential algebra over the field $\mathbb{K}$ that is (i) freely generated by the variables $x^{i}, y^{a}$ and $z^{a}$ whose respective Grassmann parities are related by

$$
\epsilon\left(z^{a}\right)=\epsilon\left(y^{a}\right)+1
$$

and (ii) endowed with the differential $\Delta$ defined via

$$
\Delta x^{i}=0, \quad \Delta y^{a}=z^{a}, \quad \Delta z^{a}=0,
$$

and the Leibnitz rule. The differential superalgebra $\mathcal{A}$ is graded by the degree of homogeneity in the variables $y^{a}$ for which the differential $\Delta$ is of degree minus one. The pairs $\left(y^{a}, z^{a}\right)$ are called contractible pairs. This terminology follows from the well-known lemma ${ }^{9}$ :

Lemma 3. The differential superalgebra $(\mathcal{A}, \Delta)$ provides a homological resolution of the polynomial algebra $\mathbb{K}\left[x^{i}\right]$. More precisely, the homology $H(\Delta, \mathcal{A})$ decomposes according to the degree of homogeneity in the variables $y^{a}$ as follows:

$$
H_{0}(\Delta, \mathcal{A})=\mathbb{K}\left[x^{i}\right], \quad H_{k}(\Delta, \mathcal{A})=0, \quad k \neq 0
$$

\section{B.2 Computation of $H(\gamma)$}

The lemma 2 combined with the rule (23) leads to the following rule for the Kronecker product

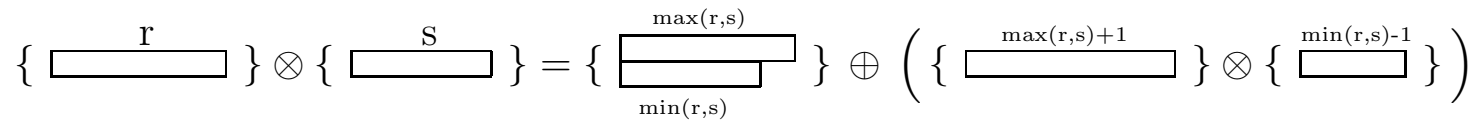

that will be very useful in the sequel.

\section{B.2.1 Unconstrained case}

The set of generators are the variables $[\Phi]$. The variables $[\varphi]$ and $[\xi]$ must be compared in the $\mathfrak{g l}(n)$-module of covariant tensors of the same rank. The covariant tensors $\partial^{k} \varphi$ belonging to the Kronecker product $\{\square \mathrm{k}$ tensors $\partial^{k+1} \xi$ belonging to the $\mathfrak{g l}(n)$-module labeled by $\{\stackrel{\mathrm{k}+1}{\square} \otimes\{\stackrel{\mathrm{s}-1}{\square}\}$. When

\footnotetext{
${ }^{9}$ For a proof, see e.g. Section 8.3 .2 of [10].
} 
doing this comparison, we remember that they are related via the formula (13) and look for a decomposition of the variables $[\Phi]$ into a set $\left\{x^{i}, y^{a}, z^{a}\right\}$ in analogy with Lemma 3.

Two distinct cases have to be addressed:

$\diamond-1 \leq k \leq s-2$ : This means that $0 \leq k+1 \leq s-1$, therefore the covariant tensors $\partial^{k+1} \xi$ decomposes as the direct sum

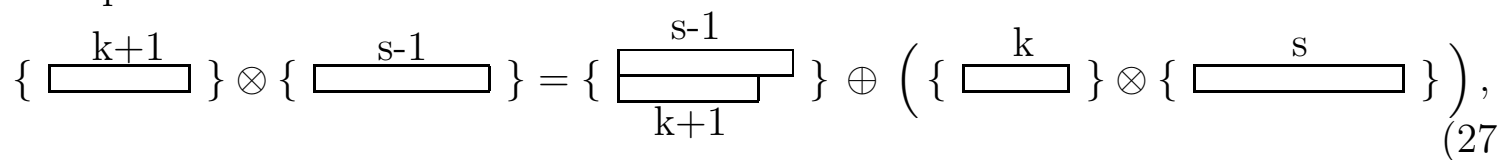

where the second term on the r.h.s. is absent for $k=-1$. The first term corresponds to irreducible covariant tensors $d^{k+1} \xi(k+1 \leq s-1)$ which are $x^{i}$ variables for the longitudinal differential $\gamma$ according to the notation of Lemma 3. The second term on the r.h.s. of (27) corresponds to covariant tensors $z^{a}$ in the Kronecker product $\{\square \mathrm{k}\} \otimes\left\{\square \mathrm{s}\right.$ \} that form contractible pairs with the variables $y^{a} \equiv \partial^{k} \varphi$ for $k \geq 0$.

$\diamond \underline{k \geq s-1:}$ The Kronecker product corresponding to $\partial^{k} \varphi$ decomposes as

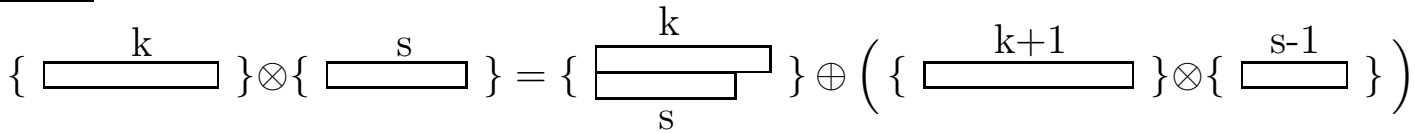

The first term on the r.h.s. corresponds to $(k-s)$ th partial derivatives of the de Wit-Freedman tensor $\partial^{k-s} \mathcal{R} \equiv x^{j}$. (The Bianchi identities are responsible for the fact that the partial derivatives of the linearized curvature tensor belongs to the irrep. labeled by two-row Young diagrams.) Eventually, the second term on the r.h.s. of (28) correspond to variables $y^{b}$ forming a contractible pair together with the variables $z^{b} \equiv \partial^{k+1} \xi$

The application of Lemma 3 is straightforward since the pairs have been explicitly separated. (The antifield variables $\left[\Phi^{*}\right]$ are inert under the action of the exterior differential.) This leads to the first part of Theorem 1.

\section{B.2.2 Constrained case: change of basis}

The basis we start with corresponds to $[\bar{\Phi}]$. The basis elements $[\widetilde{\varphi}]$ and $[\widehat{\xi}]$ must be compared in $\mathfrak{g l}(n)$-modules of covariant tensors of the same rank. To do so, we have first to apply the "converse" branching rule (25) since the fields $\bar{\Phi}$ satisfy some trace constraints. Firstly, $\widetilde{\varphi}$ are double-traceless covariant tensors of rank $s$, thus they span the direct $\operatorname{sum}[\stackrel{\mathrm{s}}{\square} \oplus \stackrel{\mathrm{s}-2}{\square}$ ] of irreducible $\mathfrak{o}(n)$-modules, which may also be labeled by $\{\square$ S $\} \ominus\{\stackrel{\mathrm{s}-4}{\square}\}$. Secondly, $\widehat{\xi}$ are traceless covariant tensors of rank $s-1$, thus they span the irreducible $\mathfrak{o}(n)$-module labeled by $[\stackrel{\mathrm{S}-1}{\square}] \uparrow\{\stackrel{\mathrm{S}-1}{\square}\} \ominus\{\stackrel{\mathrm{S}-3}{\square}\}$.

Consequently, the comparison between the tensors $\partial^{k} \widetilde{\varphi}$ and $\partial^{k+1} \widehat{\xi}$ is more involved in the constrained approach. Actually, it is practical to consider the gauge fields as unconstrained and impose the constraints separately. More specifically, when $k \geq 2$, it turns out to be convenient (and of physical significance) to split the basis elements $\partial^{k} \widetilde{\varphi}$ into the tensors $\widehat{\partial}^{k} \varphi$ and $\partial^{k-2} \mathcal{F}$ subject to the constraints $\widehat{\partial^{k}} \varphi^{\prime \prime}=0$. Let us now explain what is meant by this splitting and how it is performed. 
- To start with, we consider the product of $k$ partial derivatives $\partial_{\mu_{1}} \ldots \partial_{\mu_{k}}$ as covariant tensor in the irreducible $\mathfrak{g l}(n)$-module labeled by $\{\mathrm{k}$, which can be reduced to the sum of its traceless part $\widehat{\partial}^{k}$ labeled by $\{\square \mathrm{k}\} \ominus\{\stackrel{\mathrm{k}-2}{\square}\} \downarrow[\square \mathrm{k}$ ] and a part proportional to its trace $\square \partial^{k-2}$ labeled by $\{\stackrel{\mathrm{k}-2}{\square}$ \}.

- The space spanned by the tensors $\partial^{k} \widetilde{\varphi}$ is by definition exactly the same as the space spanned by the tensors $\partial^{k} \varphi$ quotiented by the space spanned by $\partial^{k} \varphi^{\prime \prime}$ that is labeled by the Kronecker product $\{\stackrel{\mathrm{k}}{\square}\} \otimes\{\stackrel{\mathrm{s}-4}{\square}$.

- Then we perform the following invertible change of basis $\left\{\partial^{k} \varphi\right\} \longleftrightarrow\left\{\widehat{\partial^{k}} \varphi, \partial^{k-2} \square \varphi\right\}$. Moreover, it is possible to perform a triangular invertible linear change of variables $\square \varphi \longleftrightarrow \mathcal{F}$ relating the Laplacian of the gauge field to the Fronsdal tensor (4).

- Putting all these remarks together, we have proved that the span of $\left\{\partial^{k} \widetilde{\varphi}\right\}$ is the quotient of the span $\left\{\widehat{\partial^{k}} \varphi, \partial^{k-2} \mathcal{F}\right\}$ divided by the span of $\left\{\partial^{k} \varphi^{\prime \prime}\right\}$.

To summarize, we must compare the following four Kronecker products

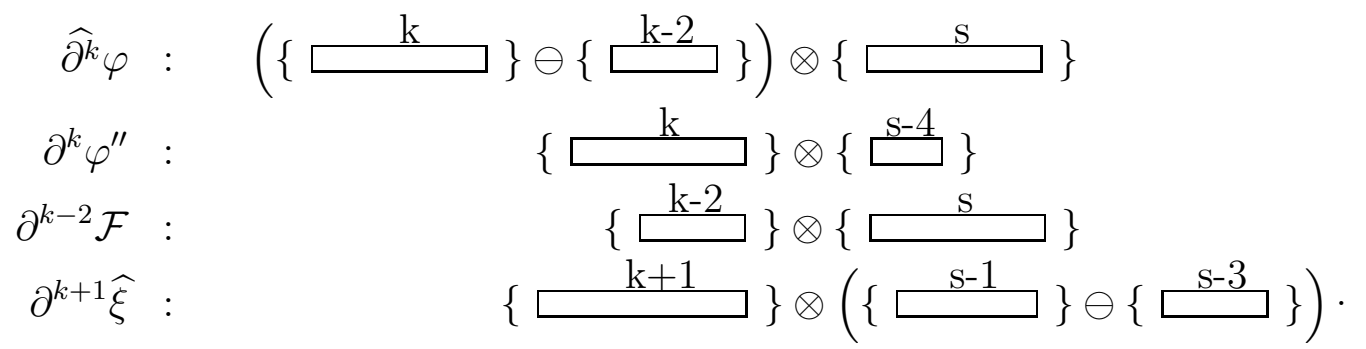

\section{B.2.3 Constrained case: decomposition of Kronecker products}

Three cases have to be distinguished:

* $-1 \leq k \leq s-4$ : The Kronecker product corresponding to the $(k+1)$ th partial derivative of the trace constraint on the ghost $\xi$ can be reexpressed as the direct sum

$$
\begin{aligned}
&\{\stackrel{\mathrm{k}+1}{\longleftarrow}\} \otimes\{\stackrel{\mathrm{s}-3}{\longleftarrow}\}=\{\stackrel{\mathrm{s}-3}{\mathrm{k}+1}\} \oplus\{\stackrel{\mathrm{s}-2}{\mathrm{k}}\} \oplus\{\stackrel{\mathrm{s}-1}{\mathrm{k}-1}\} \\
& \oplus(\{\mathrm{k}-2 \\
&\stackrel{\mathrm{s}}{\leftrightarrows}\}),
\end{aligned}
$$

where this equation is obtained by three successive application of the rule (26) when $k \geq 2$. (If $k<2$ then the formula (33) remains valid but the terms that would not be well-defined are absent.)

Combining (27) with (33) leads to the following decomposition of the Kronecker product (32):

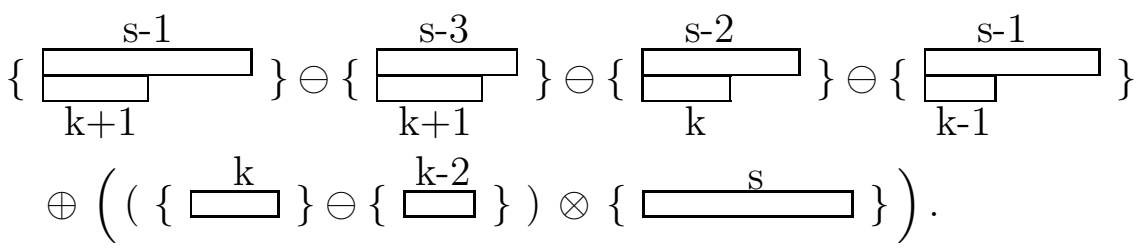


Therefore the span of tensors $\partial^{k+1} \widehat{\xi}$ when $k+1$ ranges from 0 till $s-1$ contains the irreducible $\mathfrak{o}(n)$-modules labeled by

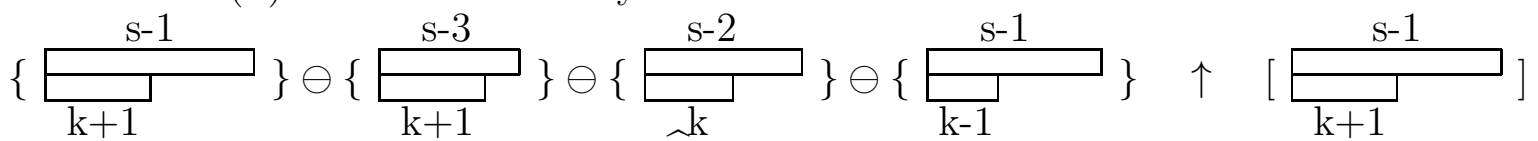
corresponding to the set of variables $\{\widehat{\xi}\}$ introduced in Subsection 4.1. The complement of the space spanned by the variables $\{\widehat{\xi}\}$ is the module labeled by the diagram in the second line of (34) the basis elements of which form contractible pairs together with the tensors $\widehat{\partial^{k}} \varphi$ described in (29).

The basis elements of the jet space that remain are therefore in the cohomology since they cannot be associated with any derivative of ghost. They are the derivatives $\partial^{k-2} \mathcal{F}$ (31) of the Fronsdal tensor subject to the constraints $\partial^{k} \varphi^{\prime \prime}=0(30)$.

$* s-3 \leq k \leq s+1$ : This case contains five subcases each of which must be treated separately. The final result reproduces the corresponding general results of the two other cases, so we leave the explicit check as an exercise for the reader.

$* k \geq s+2$ : This is equivalent to $k-2 \geq s$ therefore we apply the rule (26) three times on the Kronecker product (31), associated with the derivatives $\partial^{k-2} \mathcal{F}$ of the Fronsdal operator, and get as a result

$$
\begin{aligned}
& \{\stackrel{\mathrm{k}-2}{\square}\} \otimes\{\stackrel{\mathrm{s}}{\square}\}=\{\stackrel{\mathrm{k}-2}{\square}\} \oplus\left\{\frac{\mathrm{k}-1}{\mathrm{~s}-1}\right\} \oplus\{\underset{\mathrm{s}-2}{\rightleftarrows} \\
& \oplus(\{\stackrel{\mathrm{k}+1}{\longleftarrow}\} \otimes\{\stackrel{\mathrm{s}-3}{\square}\}) \text {. }
\end{aligned}
$$

The Damour-Deser identity relates the trace of the curvature tensor to $s-2$ curls of the Fronsdal tensor $\mathcal{R}^{\prime} \propto d^{s-2} \mathcal{F}[7,9]$. Consequently, the right-hand-side of the first line of (35) can be expressed entirely in terms of derivatives of the trace $\mathcal{R}^{\prime}$ of the Riemann tensor. Combining (35) with (28) we get that the $k$ th derivatives of the double-traceless gauge field of rank $s$ decompose as follows

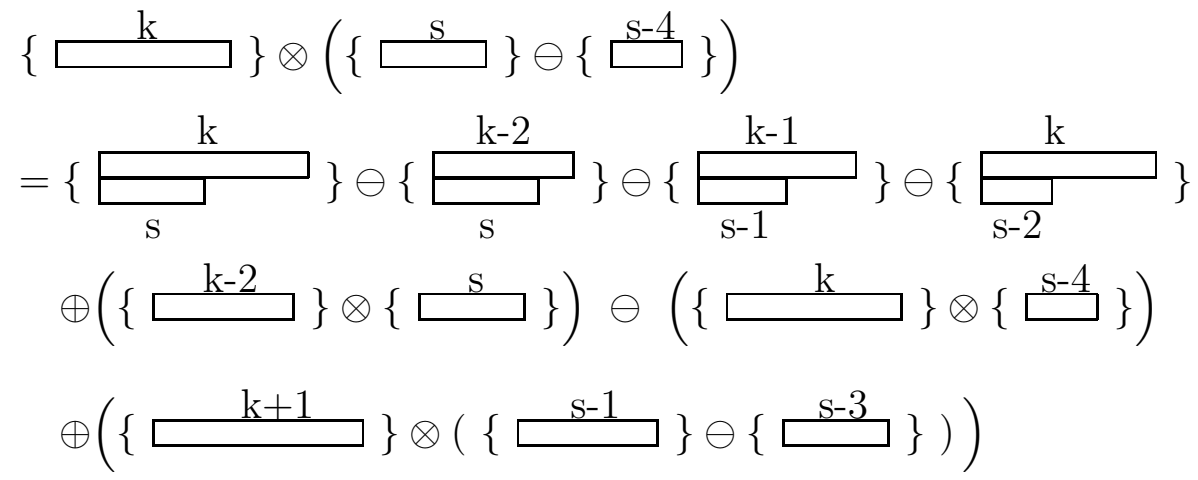

The last line of (36) is paired with the ghost sector (32) which disappear from the cohomology. The second line of (36) states that the traceless component $\widehat{[\mathcal{R}]}$ of the derivatives of the curvature tensors are in the cohomology since they span the irreducible $\mathfrak{o}(n)$-modules labeled by [ ${ }_{\mathrm{S}}^{\mathrm{k}}$ ] while the third line of (36) together with (30) and (31) show that the derivatives of the Fronsdal tensor depending on $\widetilde{\varphi}$ complete the generators of the cohomology of $\gamma$. 


\section{Proof of Theorem 2}

\section{C.1 Unconstrained off-shell Killing tensor fields}

If $\varepsilon_{\mu_{1} \ldots \mu_{s-1}}(x)$ is a formal power series in $x$, then, by definition,

$$
\varepsilon_{\mu_{1} \ldots \mu_{s-1}}(x)=\sum_{t=0}^{\infty} \lambda_{\mu_{1} \ldots \mu_{s-1}, \nu_{1} \ldots \nu_{t}} x^{\nu_{1}} \ldots x^{\nu_{t}},
$$

where the tensors $\lambda_{\mu_{1} \ldots \mu_{s-1}, \nu_{1} \ldots \nu_{t}}$ are all constant and each set of indices is symmetrized. If one acts with $s-1$ partial derivatives on both sides of the off-shell Killing-like equation (2), then the resulting equation is equivalent to

$$
\partial_{\nu_{1}} \ldots \partial_{\nu_{s}} \varepsilon_{\mu_{1} \ldots \mu_{s-1}}=0
$$

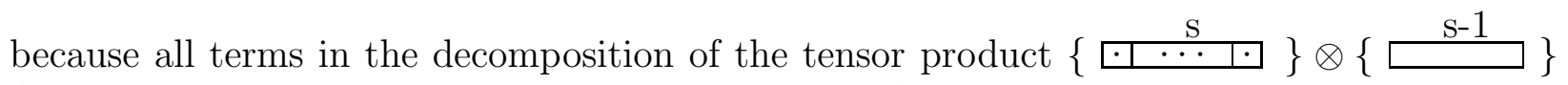
(where a dot in a box stands for a partial derivative) always contain, as a subdiagram, the Young diagram $\mathrm{S}$. corresponding to the left-hand-side of (8). In other words, the left-hand-side of (38) depends linearly on the left-hand-side of (8). Inserting (37) in (38) leads to the fact that the tensors $\lambda_{\mu_{1} \ldots \mu_{s-1}, \nu_{1} \ldots \nu_{t}}$ are zero for $t \geq s$. Then, substituting the resulting polynomial for $\varepsilon_{\mu_{1} \ldots \mu_{s-1}}$ in the original Killing-like equation (8) gives the system of equations

$$
\lambda_{\left(\mu_{1} \ldots \mu_{s-1}, \nu_{1}\right) \nu_{2} \ldots \nu_{t}}=0 \quad(t<s)
$$

which implies that the constant tensor $\lambda_{\mu_{1} \ldots \mu_{s-1}, \nu_{1} \ldots \nu_{t}}$ belongs to the $\mathfrak{g l}(n)$-module labeled by $\{\longleftarrow t-1\}$. This proves Lemma 1 .

Analogously, the point (i) of Theorem 2 is a straightforward consequence of the relations (16) applied to any monomial of the schematic form $\mathrm{M}^{t} \mathrm{P}^{s-1-t}$ which leads to a result identical to the previous one in terms of irreducible $\mathfrak{g l}(n)$-modules. The main point is that the $\mathfrak{g l}(n)$ irreducibility conditions (16) are expressed in the "antisymmetric" convention for Young diagrams. Alternatively, the isomorphism (i) follows from the fact that the algebra of Killing tensors on any constant curvature space is generated by the corresponding Killing vectors [15] (which are of course in one-to-one correspondence with the generators of the isometry algebra).

\section{C.2 Constrained on-shell Killing tensor fields}

The proof of the point (ii) of Theorem 2 requires more work and uses some general results on local Koszul-Tate cohomology groups. Let us consider the on-shell (also called "weak") Killing-like equation (9) for the constrained free spin- $s$ gauge field theory:

$$
\partial_{\left(\mu_{1}\right.} \widehat{\varepsilon}_{\left.\mu_{2} \ldots \mu_{s}\right)} \approx 0 .
$$

As explained in the previous subsection, if one acts with $s-1$ partial derivatives on both sides of (40), then the resulting equation is equivalent to ${ }^{10}$

$$
\partial_{\nu_{1}} \ldots \partial_{\nu_{s}} \widehat{\varepsilon}_{\mu_{1} \ldots \mu_{s-1}} \approx 0
$$

\footnotetext{
${ }^{10}$ The equation (41) was conjectured for arbitrary spin $s$ in [29].
} 
because the partial derivative preserves the stationary surface since the Fronsdal equation (5) does not depend explicitly on $x$.

An application of the general theorems 6.2 and 6.4 of [17] to the constrained free spin- $s$ gauge field theory of Fronsdal leads to the fact that the "characteristic cohomology group" in form degree zero is represented by the constants (see for instance $[31,32,33]$ ). In other words,

$$
\partial_{\mu} f(x,[\widetilde{\varphi}]) \approx 0 \Longleftrightarrow f(x,[\widetilde{\varphi}]) \approx c s t
$$

where "cst" stands for a constant independent of $x$ and the (partial derivatives of the) gauge field $\widetilde{\varphi}$. Therefore, (41) is equivalent to

$$
\partial_{\nu_{1}} \ldots \partial_{\nu_{s-1}} \widehat{\varepsilon}_{\mu_{1} \ldots \mu_{s-1}} \approx \lambda_{\mu_{1} \ldots \mu_{s-1}, \nu_{1} \ldots \nu_{s-1}}
$$

where the tensor $\lambda_{\mu_{1} \ldots \mu_{s-1}, \nu_{1} \ldots \nu_{s-1}}$ is constant, each set of indices is symmetrized and the indices $\mu$ are traceless. Consequently, the tensor

$$
\widehat{\varepsilon}_{\mu_{1} \ldots \mu_{s-1}}^{\prime}:=\widehat{\varepsilon}_{\mu_{1} \ldots \mu_{s-1}}-\lambda_{\mu_{1} \ldots \mu_{s-1}, \nu_{1} \ldots \nu_{s-1}} x^{\mu_{1}} \ldots x^{\mu_{s-1}}
$$

satisfies

$$
\partial_{\nu_{1}} \ldots \partial_{\nu_{s-1}} \widehat{\varepsilon}_{\mu_{1} \ldots \mu_{s-1}}^{\prime} \approx 0
$$

By repeating the previous argument $s-1$ times, we arrive at the conclusion that

$$
\widehat{\varepsilon}_{\mu_{1} \ldots \mu_{s-1}}(x,[\widetilde{\varphi}]) \approx \sum_{t=0}^{s-1} \lambda_{\mu_{1} \ldots \mu_{s-1}, \nu_{1} \ldots \nu_{t}} x^{\nu_{1}} \ldots x^{\nu_{t}}
$$

where the tensors $\lambda_{\mu_{1} \ldots \mu_{s-1}, \nu_{1} \ldots \nu_{t}}$ are constant, symmetric in each set of indices and traceless in the indices $\mu$. Substituting (42) in the (weak) Killing-like equation (40), we obtain

$$
\sum_{t=0}^{s-2}(t+1) \lambda_{\left(\mu_{1} \ldots \mu_{s-1}, \mu_{s}\right) \nu_{1} \ldots \nu_{t}} x^{\nu_{1}} \ldots x^{\nu_{t}} \approx 0 .
$$

Taking into account the fact that the left-hand-side of the (weak) equality (43) depends only on the space-time variable $x$ while the Fronsdal equation (5) is linear in the jet space variables $[\widetilde{\varphi}]$, we arrive at the conclusion that the constant tensors $\lambda$ satisfy the (strong) conditions (39).

To end up the proof of the last point of Theorem 2, we use the general property that tensors irreducible under $\mathfrak{g l}(n)$ and traceless in the indices corresponding to the largest row are traceless in all their indices (see also Lemma 4.5 of [24] for another proof of the symmetry properties of traceless Killing tensors).

\section{References}

[1] C. Fronsdal, Phys. Rev. D 18 (1978) 3624.

[2] X. Bekaert, S. Cnockaert, C. Iazeolla and M.A. Vasiliev, "Nonlinear Higher Spin Theories in Various Dimensions", in the proceedings of the First Solvay Workshop on HigherSpin Gauge Theories (Brussels, Belgium, May 2004) [hep-th/0503128]. 
[3] B. de Wit and D. Z. Freedman, Phys. Rev. D 21 (1980) 358.

[4] M. A. Vasiliev, Sov. J. Nucl. Phys. 32 (1980) 439 [Yad. Fiz. 32 (1980) 855]; Fortsch. Phys. 35 (1987) 741;

V. E. Lopatin and M. A. Vasiliev, Mod. Phys. Lett. A 3 (1988) 257.

[5] D. Francia and A. Sagnotti, Phys. Lett. B 543 (2002) 303 [hep-th/0207002].

[6] D. Sorokin, "Introduction to the classical theory of higher spins", in the proceedings of XIX Max Born Symposium (Wroclaw, Poland, October 2004) pp. 172-202 [hep-th/0405069];

N. Bouatta, G. Compere and A. Sagnotti, "An introduction to free higher-spin fields", in the proceedings of the First Solvay Workshop on Higher-Spin Gauge Theories (Brussels, Belgium, May 2004) [hep-th/0409068].

[7] T. Damour and S. Deser, Annales Poincaré Phys. Théor. 47 (1987) 277.

[8] P.J. Olver, "Differential hyperforms I", Univ. of Minnesota report 82-101;

M. Dubois-Violette and M. Henneaux, Lett. Math. Phys. 49 (1999) 245 [math.qa/9907135]; Commun. Math. Phys. 226 (2002) 393 [math.qa/0110088].

[9] X. Bekaert and N. Boulanger, Phys. Lett. B 561 (2003) 183 [hep-th/0301243]; "Mixed symmetry gauge fields in a flat background", in the proceedings of the International Seminar on Supersymmetries and Quantum Symmetries "SQS 03" (Dubna, Russia, July 2003) [hep-th/0310209].

[10] M. Henneaux and C. Teitelboim, Quantization of Gauge Systems (Princeton University Press, 1992).

[11] X. Bekaert, N. Boulanger and S. Cnockaert, in preparation.

[12] X. Bekaert and N. Boulanger, Commun. Math. Phys. 245 (2004) 27 [hep-th/0208058]; Class. Quantum Grav. 20 (2003) S417 [hep-th/0212131].

[13] O. V. Shaynkman and M. A. Vasiliev, Theor. Math. Phys. 123 (2000) 683 [Teor. Mat. Fiz. 123 (2000) 323] [hep-th/0003123]; Theor. Math. Phys. 128 (2001) 1155 [Teor. Mat. Fiz. 128 (2001) 378] [hep-th/0103208];

O. A. Gelfond and M. A. Vasiliev, [hep-th/0304020].

[14] L. P. Eisenhart, Riemannian Geometry (Princeton University Press, 1966) p. 128.

[15] G. Thompson, J. Math. Phys. 27 (1986) 2693.

[16] T. Wolf, Comp. Phys. Comm. 115 (1998) 316;

R. G. McLenaghan, R. Milson and R. G. Smirnov, C. R. Acad. Sci. Paris, Ser. I 339 (2004) 621.

[17] G. Barnich, F. Brandt and M. Henneaux, Phys. Rept. 338 (2000) 439 [hep-th/0002245].

[18] M. G. Eastwood, "Higher symmetries of the Laplacian" [hep-th/0206233]. 
[19] M. A. Vasiliev, Phys. Lett. B 567 (2003) 139 [hep-th/0304049].

[20] F. A. Berends, G. J. H. Burgers and H. van Dam, Nucl. Phys. B 271 (1986) 429.

[21] S. R. Coleman and J. Mandula, Phys. Rev. 159 (1967) 1251;

R. Haag, J. T. Lopuszanski and M. Sohnius, Nucl. Phys. B 88, 257 (1975).

[22] X. Bekaert, in preparation.

[23] M. Henneaux, Nucl. Phys. Proc. Suppl. 18A (1990) 47.

[24] G. Barnich, M. Grigoriev, A. Semikhatov and I. Tipunin, "Parent field theory and unfolding in BRST first-quantized terms" [hep-th/0406192].

[25] A. Mikhailov, "Notes On Higher Spin Symmetries" [hep-th/0201019].

[26] A. Sagnotti, E. Sezgin and P. Sundell, "On higher spins with a strong $S p(2, \mathbb{R})$ condition", in the proceedings of the First Solvay Workshop on Higher-Spin Gauge Theories (Brussels, Belgium, May 2004) [hep-th/0501156].

[27] D. Anselmi, Nucl. Phys. B 541 (1999) 323 [hep-th/9808004]; Class. Quantum Grav. 17 (2000) 1383 [hep-th/9906167];

M. A. Vasiliev, "Higher spin gauge theories: Star-product and AdS space", [hep-th/9910096] in M. Shifman ed., The many faces of the superworld (World Scientific, 2000) Section 2.

[28] M. A. Vasiliev, "Actions, charges and off-shell fields in the unfolded dynamics approach" [hep-th/0504090].

[29] N. Bouatta, "Approche BRST des systèmes invariants de jauge à spins élevés", Master Degree Thesis defended at the Free University of Brussels (Academic year 2003-2004).

[30] G. Barnich, private communication.

[31] M. Henneaux and B. Knaepen, Nucl. Phys. B 548 (1999) 491 [hep-th/9812140].

[32] N. Boulanger, T. Damour, L. Gualtieri and M. Henneaux, Nucl. Phys. B 597 (2001) 127 [hep-th/0007220].

[33] J. A. Garcia and B. Knaepen, Phys. Lett. B 441 (1998) 198 [hep-th/9807016];

X. Bekaert, N. Boulanger and M. Henneaux, Phys. Rev. D 67 (2003) 044010 [hep-th/0210278];

N. Boulanger and S. Cnockaert, JHEP 0403 (2004) 031 [hep-th/0402180];

X. Bekaert, N. Boulanger and S. Cnockaert, J. Math. Phys. 46 (2005) 012303 [hep-th/0407102].

[34] D.E. Littlewood, The theory of group characters (Clarendon, 1940); G. R. E. Black, R. C. King and B. G. Wybourne, J. Phys. A: Math. Gen. 16 (1983) 1555; S. A. Fulling, R. C. King, B. G. Wybourne and C. J. Cummins, Class. Quantum Grav. 9 (1992) 1151.

[35] R.C. King, J. Phys. A: Math. Gen. 8 (1975) 429. 